\title{
Comparison of women with cervical human papillomavirus infection and genital warts. I. Some behavioural factors and clinical findings
}

\author{
D Hellberg, N Borendal, B Sikström, S Nilsson, P-A Mårdh
}

\begin{abstract}
Objective-To determine if behavioural factors, other than sexual, differ between women with cervical human papillomavirus infection (CHPI) and those with genital warts (GW).

Design-A structured, personal in-depth interview which included details on sexual behaviour, hygiene, gynaecological complaints, and demographical characteristics and a gynaecological examination which included vaginal culture and human papillomavirus (HPV) typing with Southern blot.
\end{abstract}

Subjects-Women ( $\mathrm{n}=972)$ who had attended two family planning clinics and one youth clinic for contraceptive advice. Results-Out of this female population 66 $(6 \cdot 8 \%)$ were found to have CHPI, 39 $(4 \cdot 0 \%)$ had genital warts (GW), and 30 $(3 \cdot 1 \%)$ women reported genital warts within the last two years. The women with CHPI had had significantly less education, were more often immigrants, had a vaginal flora change more frequently and were more often smokers than the women with $G W$. The women with $G W$ reported lower abdominal pain significantly more often, had a leucocyte dominance in the vaginal secretion more often and favoured bathing in a bathtub more than the women with CHPI. After adjustment for sexual behavioural factors the significant difference between the two groups for bathing in a bathtub and lower abdominal pain remained.

Conclusion-Although both conditions are caused by HPV, there are behavioural differences between women with CHPI and women with CA.

WHO Collaborative Centre for STD and their Complications, Uppsala University, Uppsala, and Departments of Obstetrics and Gynaecology, Falun Hospital, Falun, and Motala Hospital, Motala, Sweden D Hellberg $\mathrm{N}$ Borendal B Sikström $S$ Nilsson P-A Mårdh

Correspondence to: Dr Dan Hellberg. Dr Dan Hellberg,
Department of Obstetrics and Gynaecology, Falun Hospital, S-791 82 Falun, Sweden.

Accepted for publication 11 November 1994

\section{Introduction}

Some 70 types of human papillomavirus (HPV) have been identified. Of approximately 25 types affecting the cervical mucosa, ${ }^{1}$ types $16,18,31,33$ and 35 are the most frequent; all of these have high oncogenic potential. ${ }^{12}$ Latent HPV infections can be detected by DNA hybridisation. Subclinical infections may be seen as flat condylomas on colposcopic examination. One clinical manifestation of genital HPV infection is benign genital warts (GW), condyloma acuminata. GW are almost exclusively caused by HPV 6 and 11 , both types have low oncogenic potential.

An association between GW and cervical dyskaryosis, as well as the role of HPV in cervical cancer in situ and in invasive cancer, has been demonstrated. ${ }^{23}$

Although caused by the same virus, the two conditions are different entities. It is not known whether CHPI and GW affects the same group of women, or if they affect women with similar behavioural characteristics other than sexual risk behaviour.

This study compares some behavioural factors, such as hygiene and smoking, in women with a cervical HPV infection and in women with genital warts, who attended two family planning clinics and one youth clinic for contraceptive advice. The study also compares genital symptoms and signs and vaginal flora in women with GW and in women with CHPI.

\section{Material and methods}

Between November 1989 and January 1991 1011 women were recruited to the study at the family planning clinic and youth clinic at Eskilstuna Hospital and at the family planning clinic at Danderyds Hospital, Stockholm. Eskilstuna is a medium-sized town outside Stockholm, with a population of approximately one million.

A selected number of midwives were specially trained for the particular tasks involved in the study. Women, with certain, predetermined positions on the out-patients list, were asked by the midwife if she agreed to enter the study. The midwives were not involved in the time-booking, so the identity of the women was unknown to the midwives. Patients were added to the out-patient list consecutively when the patient booked a time, so as to avoid selection bias.

One thousand and eleven (93.9\%) out of 1077 who were asked, agreed to participate. HPV-typing was performed on 972 (96.1\%) of the women.

The midwives conducted the approximately one to one and a half hour long structured personal interviews. Before the interview a short questionnaire was handed out as a self-administered form for questions of non-controversial nature, which the woman completed after the interview. The midwives also collected samples for laboratory testing.

Anonymity was guaranteed, to ensure as honest answers as possible. No details that could identify the woman were neither included in the patient record form, nor in 
computerised data. Only the authors had access to the patients' codes.

The interview included 296 questions. Details about genital symptoms, social and demographic characteristics, smoking and alcohol use and drug abuse, genital hygienic practice and some questions concerning the male partners, were included.

Before vaginal specimens were collected, a careful gynaecological examination was made, noting the character of the vaginal secretion, bleedings, fissures, ulcerations and vesicles, appearance of the genital mucosal surface, possible ectopies and warts (before and after application of acetic acid). Vaginal cultures were made for Candida spp., Lactobacilli spp. and anaerobic and facultative anaerobic bacteria.

Wet smear microscopy from the lateral and posterior vaginal fornix was used for analysis of vaginal epithelial cell/leucocyte ratio, trichomonades, bacteria with mobiluncus morphotype and clue cells. Bacterial vaginosis (BV) was defined as three criteria out of; grey homogenous discharge, clue cells, a vaginal $\mathrm{pH}>4.7$ and a positive amine test. ${ }^{4}$

For detection and typing of HPV-DNA, cell samples were collected from the ectocervix by means of a brush device (Cytobrush, Medscand, Malmö, Sweden) and washed off with $1 \mathrm{ml}$ aqueous lytic buffer (Oncor ${ }^{R}$ Inc., Gaithersburg, $M D$, USA) in a $2 \mathrm{ml}$ microfuge tube. ${ }^{5}$ The tubes were mailed to the laboratory at ambient temperature and stored at $4^{\circ} \mathrm{C}$ until analysed. HPV-typing was made by Southern blot. Sodium dodecyl sulphate was added to the specimens whereafter they were digested with proteinase $\mathrm{K}$, incubated at $50^{\circ} \mathrm{C}$ for 15 minutes. Residual peptides were salted out by adding a "protein precipitating agent" $\left(\right.$ Oncor $\left.^{\mathrm{R}}\right)$ retaining the supernatant after vortexing and centrifugation. The DNA was then precipitated from this supernatant with 3 volumes of ethanol at room temperature. After careful washings twice with ethanol, with resultant DNA pellets, the preparations were dissolved overnight at $52^{\circ} \mathrm{C}$, using TrisEDTA buffer. After digestion with two restriction enzymes (Bam $\mathrm{Hl}$ and Pst 1) and electrophoresis in $1 \%$ agarose gel the separated DNA fragments were first depyrinated with hydrochloric acid, denatured with sodium hydroxide whereafter the blotting on to a nylon membrane was obtained with limited vacuum using the Probe Tech electrophoresis and transfer equipment $\left(\right.$ Oncor $\left.^{\mathrm{R}}\right)$. The hybridisation was then performed using a mixture of subgenomic probes selected to label only one band of a defined size for each of the HPV types studied (Oncor Human Papillomavirus Typing System ${ }^{R}$ ). Cases where a distinct band was obtained, however unrelated to any of the standards, were designated as unknown types.

The material was computerised and analysed with JMP statistical program. ${ }^{6}$ Initial significance tests were done with chi square for nominal variables (Pearson and likelihood ratio) and $t$ test for continuous variables. In order to assess the simultaneous effect of more than one variable, multi-way frequency tables were analysed by means of logistic regression (analysis of $\log$ likelihood), so as to identify and check for possible confounding.

\section{Results}

There were 66 women (6.8\%) with CHPI. HPV type 6 was found in four women, HPV 11 in four, HPV 16 in 25, HPV 18 in 10, HPV 31 in 14, HPV 33 in 5, HPV 35 in five, while in eight women nontypable isolates were found. Nine women were infected by more than one HPV-type. Three of these were concomitant HPV type 16 and 31 infections, while the remaining six infections with more than one HPV-type were combinations of different types.

Thirty-nine $(4.0 \%)$ women had current GW. Another 30 women (3.1\%) reported treatment of GW within the last two years. Those 69 women were used as GW cases in the analyses of behavioural factors. For current symptoms and findings, only current $\mathrm{GW}$ was compared with CHPI.

Three out of the 66 females $(4 \cdot 6 \%)$ with CHPI also had current GW ("double infection" in the following). The difference was nonsignificant ( $p=0.82$ ), as compared to the frequency of $\mathrm{GW}$ in remaining study population. After exclusion of the three cases with double infections, the CHPI group consisted of 63 and the GW group with current disease of 36 females. Mean age for the CHPI cases was 24.5 years, and for the GW cases $26 \cdot 8$ years $(p=0 \cdot 10)$. All comparisons between the two groups are in the following adjusted for the age difference.

After exclusion of seven women with double infections in the CHPI group and in those with current $\mathrm{GW}$, or with $\mathrm{GW}$ within the last two years, these two groups consisted of 59 and 62 women, respectively.

The mean age in these latter two groups was similar $(24.8$ years vs. 25.6 years, $p=$ 0.49 ).

The women with CHPI had less often education above primary school $(p=0.05)$, were more often immigrants of the first or second generation $(p=0.02)$ and were more frequently smokers $(p=0.04)$ than the females with GW (table 1). When age, education and smoking were evaluated in multifactorial analyses, where adjustment was made for age, age at first intercourse and the number of lifetime sexual partners, the differences between the two groups were no longer significant.

Table 1 Some characteristics in women with cervical human papillomavirus infection (CHPI) and among women with previous or current genital warts $(G W)$

\begin{tabular}{llll}
\hline & $\begin{array}{l}\text { CHPI (\%) } \\
n=59\end{array}$ & $\begin{array}{l}G W(\%) \\
n=62\end{array}$ & $p^{*}$ \\
\hline Steady partner & $39(66 \cdot 1)$ & $21(66 \cdot 1)$ & 0.99 \\
Smoker & $31(52 \cdot 5)$ & $21(33.9)$ & 0.04 \\
Regular drinker of alcohol & $42(71 \cdot 2)$ & $38(61 \cdot 3)$ & 0.25 \\
Higher education than & & & \\
$\quad \begin{array}{l}\text { Elementary school } \\
\text { Immigrant }\end{array}$ & $35(59 \cdot 3)$ & $47(75 \cdot 8)$ & 0.05 \\
Bathing in bathtub & $15(25 \cdot 4)$ & $6(9 \cdot 7)$ & 0.02 \\
& $34(57 \cdot 6)$ & $50(80 \cdot 7)$ & 0.006 \\
\hline
\end{tabular}

*All $p$ values adjusted for age. 
Table 2 Genital symptoms and signs in women with cervical human papillomavirus infection (CHPI) and women with current genital warts (GW)

\begin{tabular}{lccl}
\hline & CHPI $(\%)$ & $G W(\%)$ & \\
& $n=63$ & $n=36$ & $p^{\star}$ \\
\hline Lower abdominal pain & $27(42.9)$ & $22(61 \cdot 1)$ & 0.05 \\
Dysmennorhea & $27(42.9)$ & $22(61 \cdot 1)$ & 0.09 \\
Dysuria & $7(11 \cdot 1)$ & $7(19 \cdot 4)$ & 0.09 \\
Fishy vaginal odour & $9(14 \cdot 3)$ & 0 & 0.86 \\
Fissures, genital mucosa & $6(9 \cdot 5)$ & 0 & 0.89 \\
Abnormal vaginal discharge & $25(45 \cdot 5)$ & $14(46 \cdot 7)$ & 0.88 \\
Bacterial vaginosis & $13(22 \cdot 0)$ & $5(14 \cdot 3)$ & 0.33 \\
\hline
\end{tabular}

$\star$ All $p$ values adjusted for age.

Table 3 Wet smear in women with cervical human papillomavirus infection (CHPI) and in women with current genital warts (GW)

\begin{tabular}{llrl}
\hline & $\begin{array}{l}\text { CHPI }(\%) \\
n=63\end{array}$ & $\begin{array}{l}G W(\%)^{\star} \\
n=36\end{array}$ & $p$ \\
\hline Leukocytes > & & & \\
vaginal epithelial cells & $12(20 \cdot 3)$ & $14(40 \cdot 0)$ & 0.06 \\
Clue cells & $20(31 \cdot 8)$ & $6(16 \cdot 7)$ & $0 \cdot 13$ \\
Amine test, positive & $21(33.9)$ & $8(22 \cdot 9)$ & $0 \cdot 18$ \\
Vaginal pH > 4.7 & $21(33.9)$ & $11(32 \cdot 4)$ & 0.89 \\
\hline
\end{tabular}

*All $p$ values adjusted for age.

The females with GW favoured bathing in a bathtub more than those with CHPI. The latter correlation was significant $(p=0.01)$ also after adjustments as described above.

The only significant difference between the CHPI and the GW groups regarding current symptoms and signs was an increased frequency of lower abdominal pain in women with GW $(p=0.05)$ (table 2). The significant difference between the two groups remained $(p=0.04)$ after adjustment for sexual factors, as mentioned above. For dysmennorhoea, a significant difference diminished when adjustment for age was made. Reports of a fishy vaginal odour and fissures (table 3 ) were in the crude analyses found significantly more common among the women with CHPI than the GW cases, but the significance was lost after age-adjustment.

Analysis of vaginal wet smear (table 3) showed that leucocyte, as compared with vaginal epithelial cell, predominance was twice as frequent in the GW as in the CHPI group. After age-adjustment, the difference was only of borderline significance $(p=0.06)$.

Three of four criteria for bacterial vaginosis (BV), namely a positive amine test, $\mathrm{pH}>4 \cdot 7$ and clue cells, were numerically more often found among the CHPI cases, as compared with the GW cases, but the differences did not reach statistical significance.

\section{Discussion}

Our study showed that women with CHPI and with GW differed with regard to a number of epidemiological variables and some gynaecological findings, but that most of these differences were secondary to sexual behavioural factors.

Another finding was that there was no correlation between the females with CHPI and GW as compared with the frequency of GW in the whole population of 972 women studied.
Of gynaecological symptoms, lower abdominal pain occurred more frequently in the women with CA than in those with CHPI. The same association, although not reaching significance, was found for dysmennorhoea. In the personal interview, abdominal and menstrual problems were in separate sections and these two groups of women were not the same, but were significantly associated with each other. As the warts were generally located in the vulvar and perianal area there is no obvious explanation for this finding.

Both a history of a fishy odour of the vaginal secretion, associated with $\mathrm{BV},{ }^{7}$ and fissures, associated with HPV vulvovaginitis, ${ }^{8}$ were significantly correlated to the women in the CHPI group, as compared with the women in the GW group, in the crude analyses, but these significant differences were lost when adjusted for age. As these variables were significantly correlated to CHPI, as compared with the remaining study population (unpublished observations), there might be a difference also between the women with GW and those with CHPI. BV has in different studies both been correlated and inversely correlated to genital HPV infection. ${ }^{9}$

Almost half of all cases of CHPI and GW had an abnormal vaginal discharge, as evident on the clinical examination. This discharge was microscopically purulent in twice as many cases of GW, as compared with those with CHPI. We will in a further study report on the microbiological findings of vaginal cultures in the two compared groups.

Among previously established markers or risk factors for STDs, smoking, ${ }^{10}$ but not alcohol use $^{11}$ was significantly more frequent among the women with CHPI, than in those with GW. The smoking habits of the women with GW were equal to that of the study population of 972 women, while more than 50 per cent of the females in the CHPI group were smokers. There might be a pathogenic role for smoking in cervical HPV infections. ${ }^{12} 13$ Previous studies on smoking habits in women with $\mathrm{CHPI}^{14}$ and $\mathrm{GW}^{15}{ }^{16}$ have not given conclusive results. In STD populations, a higher smoking frequency have been reported than in comparison groups. ${ }^{17}$ Thus, any study which is aimed to explore the role of risk factors and risk markers for STDs that does not adjust for sexual and other risk factors must be considered inconclusive.

Socioeconomic status (SES) is no longer a risk factor for cervical neoplasia in Sweden. ${ }^{10}$ In the women with CHPI and with GW we analysed variables such as full-time employment, prolonged sick-leave, and unemployment, but no differences, except for education, ${ }^{9}$ between the two types of HPV infections studied were found. Thus, education seems to have replaced SES as predictor of sexual risk behaviour in Sweden.

Of genital hygienic measures studied, regular bathing in a bathtub differed between the women with GW and the CHPI cases, being favoured by the females with GW. Our finding of bathing as a risk factor, independent of sexual risk behaviour, indicates that fomites 
cannot be excluded in non-sexual transmission of HPV. GW in adolescents, who did not admit intercourse, has been reported, ${ }^{2}$ but HPV could have been transmitted through digital and oral sex. There is yet no proved non-sexual transmission of GW in adults. ${ }^{18}$

CHPI was relatively more common in first and second generation of immigrants, whereas GW was more common among Swedish natives. Although the difference was non-significant after adjustment for sexual factors one cannot exclude regional differences in the occurrence of different HPV types. ${ }^{1920}$ In our study population, CHPI occurred more frequent among females reporting casual sex on foreign journeys, independent of other risk behaviours (unpublished observations).

Our study showed that women with CHPI and women with BV differ on a number of behavioural characteristics and clinical findings. Despite adjustment for possible confounding factors, bathing in bathtub and lower abdominal pain differed significantly between cases and controls. Other differences, but not of conventional significance in multifactorial analyses were smoking, immigrant status and vaginal flora change.

1 von Krogh, G. Genitoanal papillomavirus infection: diagnostic and therapeutic objectives in the light of curren epidemiological observations. Int $\mathcal{f}$ STD AIDS 1991;2: 391-404.

2 Moscicki A-B. Human papillomavirus infections. Adv Pediatr 1992;39:257-81.

3 Franceschi S, Doll R, Gallwey J, et al. Genital warts and cervical neoplasia: An epidemiological study. Br f Cancer cervical neoplas

4 Amsel R, Totten PA, Spiegel CA, Chen KCS, Eschenbach $D$, Holmes KK. Nonspecific vaginitis. Diagnostic criteria and microbial and epidemiologic associations. $A m \mathcal{F}$ Med 1983;74:14-22.
5 Hjerpe A, Lindh E, Bistoletti P, et al. Use of cervical Cytobrush samples for dot-blot detection and Southern blot typing of human papillomaviruses using subgenomic probes. Analyt Quant Cytol Histol 1990;12. nomic prob-305.

6 SAS Institute Inc. FMP users guide. Cary, USA 1989.

7 Barbone FH, Austin WC, Alexander WJ. A follow-up study of methods of contraception, sexual activity, and rates of trichomoniasis, candidiasis, and bacterial vaginosis. Am f Obstet Gynecol 1990;163:510-4.

8 Boden E, Eriksson A, Rylander E, von Schoultz B. Clinica characteristics of papillomavirus vulvovaginitis. Acta Obstet Gynecol Scand 1988;67:147-51.

9 Moi H. Prevalence of bacterial vaginosis and its association with genital infections, inflammation, and contraceptive methods in women attending transmitted disease and primary health clinics. Int $\mathcal{F} S T D$ AIDS 1990;1:86-94

10 Hellberg D, Valentin J, Nilsson S. Smoking and cervical intraepithelial neoplasia. An association independent of other risk factors? Acta Obstet Gynecol Scand 1986;65: other ris

11 Ericksen KP, Trocki KF. Behavioral risk factors for sexually transmitted diseases in American households. Soc $\mathrm{Sc}$ Med 1992;34:843-53.

12 Greenberg J, Magder L, Aral S. Age at first coitus. A marker for risky sexual behavior in women. Sex Transm Dis 1992;19:331-4.

13 Hellberg D, Nilsson S, Haley NJ, et al. Smoking and cervical intraepithelial neoplasia: nicotine and cotinine in . Am 7 Obstet Gynecol 1988;158:910-3.

14 Ley C, Bauer HM, Reingold A, et al. Determinants of genital human papillomavirus infection in young women. ital human papillomavirus infection

15 Barton SE, Jenkins D, Cuzick J. Effect of cigarette smoking on cervical epithelial immunity: a mechanism for neoplastic change? Lancet 1988;ii:652-4.

16 Daling JR, Sherman KJ, Weiss NS. Risk factors for condy loma acuminatum in women. Sex Transm Dis 1986;13: 16-8.

17 Tyring SK, Cauda R, Baron S, Whitley RJ. Condyloma acuminatum: Epidemiological, clinical and therapeutic aspects. Eur $\mathcal{F}$ Epidemiol 1987;3:209-15.

18 Gutman LT, Herman-Giddens ME, Phelps WC Transmission of human genital papillomavirus disease: Comparison of data from adults and children. Pediatrics 1993;91:31-8.

19 Beutner KR, Becker TM, Stone KM. Epidemiology of human papillomavirus infections. Dermatol Clin 1991;9. 211-8.

20 Kjaer SK, Möller Jensen O. Comparison studies of HPV detection in areas at different risk for cervical cancer. In The Epidemiology of Cervical Cancer and Human Papillomaviruses, Munoz N, Bosch FX, Shah KV, Meheus A (eds), Lyon IARC, 1992. 